\title{
Effect of Yielding Core Length on Cyclic Behaviour of Buckling Restrained Braces
}

\author{
[ Satish Kumar S R]
}

\begin{abstract}
Buckling Restrained Braces (BRBs) have been found to perform better than conventional braces in resisting cyclic lateral loads due to earthquakes. The tendency of a brace to undergo global buckling is resisted by a sleeve tube and concrete in between. The bond between the core and concrete is broken to ensure that only the core gets loaded. This also decouples the strength from the stiffness and it is possible to vary them independent of each other. The paper describes the cyclic tests on two BRBs of identical strength but slightly different stiffness and concludes that much work needs to be done to evaluate the optimum values for a particular structure.
\end{abstract}

Keywords - Buckling restrained braces, Reduced core length

\section{Introduction}

Buckling restrained braces (BRBs) have stable and symmetrical hysteresis behavior under cyclic loading. It is achieved by a ductile steel core, encased in a steel tube with cement mortar which acts as a restraining system. An unbonding layer over the steel core eliminates shear transfer to the outer casing. With reference to Fig.1.2 which gives a schematic diagram of the mechanism of BRB. BRB Frames act as stiff reliable structural system that limits nonstructural damage and damage is concentrated in discrete replaceable brace elements. BRBs impose smaller forces on the surrounding structural elements than conventional CBFs, which is particularly advantageous considering capacity design principles. BRBs also facilitate construction of multistorey buildings, which respond with uniform damage throughout the stories and thus avoids formation of weak stories.

A BRB core can be essentially divided into three parts as shown in the Fig2.3.Part $\mathrm{C}$ represents the buckling restrained-yielding part. This segment has a reduced cross section which ensures that no yielding can take place in any other segment of the brace. It is restrained against buckling by the concrete and steel casing around it. Part B represents restrained- non-yielding part. This segment has an increased cross section which ensures no yielding takes part in this section. This part is also restrained against buckling. It also act as a transition part between A and C. Finally Part A which is the Unrestrained-Non yielding part. It houses the connection portion of the brace. It has a larger moment of Inertia than the central core by virtue of the stiffeners attached. The sleeve is a composite section of a steel tube and concrete mortar and acts as the buckling restraining mechanism. The core and the sleeve together constitute the buckling restrained brace (BRB). By varying the length of the yielding part, suitable stiffness and energy dissipation capacity can be obtained.

\section{S.R.Satish Kumar}

Indian Institute of Technology Madras, Chennai 600 036, India.
A variety of tests have been carried out on BRBs each with a specific objective of either confirming or enhancing the behaviour by using different materials for debonding, varying the contraction allowances, clearances between restraining panels, stiffening the projection beyond the sleeve and varying the length of the yielding part of the core (Iwata et al 2000, Tremblay 2004, Masoud 2011 and Seyyadet al 2014).

The advantage of varying the length of the yielding part was studied analytically by Ravikumar et al (2007). By carrying out time-history analyses on a single bay tenstoreyed building with diagonal BRBs, they showed that the length of the yielding part can be varied over the stories to get better performance of the building in terms of storey drifts and storey shears.

Masoud (2011) conducted experimental optimization studies on steel core lengths in buckling restrained braces. As the core length decreases, energy dissipation of the brace increases overall. This is easily justified since not only brace susceptibility to local and global buckling decreases, but also the core undergoes significant plastic deformations and hysteretic energy dissipation inherently increases. But susceptibility to low-cyclic fatigue is increased as plastic strains tend to accumulate. The optimized length for BRB is the shortest length that can sustain total number of required cycles according to the loading protocol provisions of a specific code. Coffin-Manson relationship was made use of for deriving the particular length.

Optimized core length, confirming to FEMA-450 loading protocol was obtained, for a frame of height $3 \mathrm{~m}$ and span $5 \mathrm{~m}$, as $100 \mathrm{~cm}$. Experimental tests on four specimens having core lengths between 60 and $130 \mathrm{~cm}$ were carried out. Braces, with core length less than the optimized length, exhibited strength deterioration noticeably sooner than the other two specimens.

Seyyedet al (2014) proposed the concept of reduced length BRBs. All-steel reduced length BRBs (RLBRBs) are designed, detailed and constructed using a special debonding and stopper mechanism. The brace with RLBRB consists of an elastic part and a yielding part. The elastic part is designed not to yield and not to buckle under the design earthquake while the fuse (RLBRB) is functioning. The lowcycle fatigue (LCF) failure and the energy dissipating capacity of RLBRB were experimentally and analytically tested. All specimens behaved well up to $5 \%$ strain and cumulative plastic deformation of maximum 666 microns was obtained (AISC specifies a minimum of 200 microns).The specimens tested withstood high axial strains of $4-5 \%$ without any global or local failure. The hysteretic responses of the specimens were stable and symmetric. Numerical models were developed and nonlinear cyclic analyses were performed to provide better insight into the core and enhancing the performance as well as application of the RLBRB in the brace configuration. 

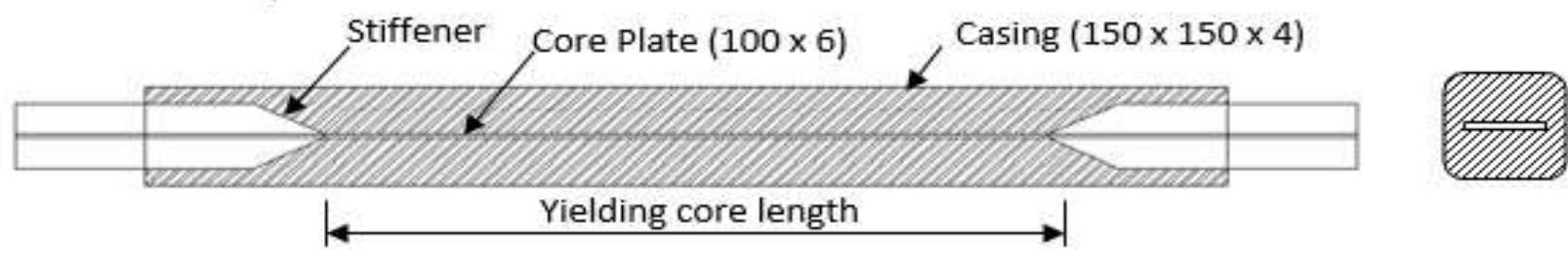

Fig. 1 BRB Specimen details

\section{Test Program}

Cyclic tests were carried out on Sub-assemblages with low-cost prototypes of buckling restrained braces. Two specimens were prepared with identical cross-sections but varying the length of the yielding core plate. The core plates were of width $100 \mathrm{~mm}$ and thickness $6 \mathrm{~mm}$ encased in square tubes of size $150 \mathrm{~mm}$ and thickness $4 \mathrm{~mm}$ as shown in Fig. 1. Both ends of the core plate were stiffened at the front and back by welding plates of size $50 \mathrm{~mm}$ and thickness $6 \mathrm{~mm}$ to get a cruciform section for the nonyielding part. The stiffeners were given transitions of slope 1:2 at their inner ends to avoid bearing on the filler concrete. To enable the core to undergo local buckling, $4 \mathrm{~mm}$ thick cardboard strips were used at the front and back. The core and stiffener, along with the cardboard was then wrapped with layers of polyethylene sheets to break the bond with the filler-concrete. The assembly was then inserted into the square tube and by closing one end of the tube, selfcompacting concrete was poured from the other end. Both specimens had a total length of $1846 \mathrm{~mm}$ but their yielding core lengths were $1000 \mathrm{~mm}$ and $1120 \mathrm{~mm}$. The yield strength of the core plates was $317 \mathrm{~N} / \mathrm{mm} 2$ giving the yield load for the braces to be $190.2 \mathrm{kN}$. The corresponding yield deformations were $1.6 \mathrm{~mm}$ and $1.8 \mathrm{~mm}$ for specimen 1 and specimen 2, respectively. The ultimate stress was $456 \mathrm{MPa}$ which is 1.44 times the yield stress. In order to simulate practical multi-bolt connections, the brace was connected at either end by six HSFG Bolts of Grade 10.9 and diameter 12 $\mathrm{mm}$.

The test set up used in the present study is similar to that used by Iwata et al (2000) and consists of a pinned-base column which is supported diagonally by the buckling restrained brace. The column was of ISMB 250 section (IS 800:2007). The column-brace connection is connected to a servo-hydraulic actuator system by means of which incremental cyclic displacements are imposed on the system. The computer controlled actuator was of capacity $1000 \mathrm{kN}$ and had a stroke of $\pm 125 \mathrm{~mm}$. Adequate lateral supports were given to ensure that the A-type truss frame system remains in its own plane throughout the test. Strain gauges pasted in the yielding part of the core and LVDTs were used to monitor strains and displacements in the brace. Schematic diagram of the test setup is given in Fig.2and a photo is shown in Fig. 3.

\section{Test Results}

Specimen 1 was first loaded on the tension side up to the yield displacement (see Fig. 4). In the second cycle, a maximum of $2.1 \delta y$ was reached. Unloading was with elastic stiffness but while loading on the compression side showed a rounded curve probably due to slight buckling of the core within the confine of the sleeve. Reloading on tension side showed a slightly higher yield point due to cyclic strain hardening. The second compression cycle attained the yield load. In the third cycle, displacements of $2.75 \delta y$ and $-2.94 \delta y$ were attained. The third compression cycle showed a decrease of $8 \%(\mathrm{H} / \mathrm{Hy}=0.92)$ in the strength at the yield displacement. This was partly due to kinematic hardening and partly due to further buckling of the core plate in a higher mode. In the final cycle, at $3.7 \delta y$ the strength remained at $1.2 \mathrm{Hy}$ in tension and at $-4.1 \delta \mathrm{y}$ it was $11 \mathrm{Hv}$ in comnrescion Thereafter the test was terminated

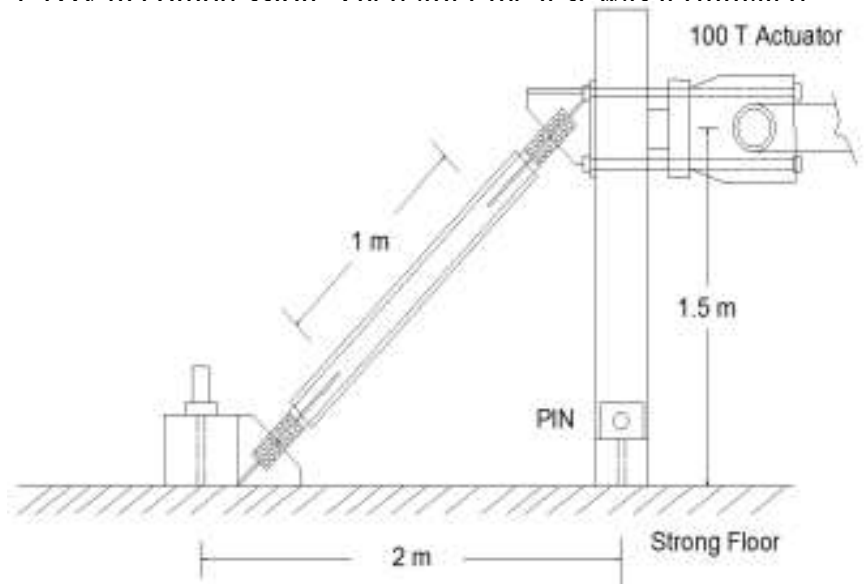

Fig. 2 Schematic diagram of test setup

Specimen 2 was also loaded on the tension side and showed a rounded curve while loading on the compression side and reached only $0.75 \mathrm{Hy}$ at $-\delta y$ (see Fig. 5). Reloading on the tension side, it again reached the yield strength and came back to $-2.2 \delta y$ by almost the same path as in the first cycle to achieve a marginally higher strength of $-1.1 \mathrm{Hy}$. In the next cycle of amplitude $2.0 \delta \mathrm{y}$, it strain hardened and achieved a strength of $1.3 \mathrm{Hy}$ although yielding started at $0.7 \mathrm{Hy}$. Unloading and reloading up to $-0.7 \mathrm{Hy}$ was almost elastic and then at $-3 \delta y$, it achieved a strength of $-1.3 \mathrm{Hy}$ thereby confirming significant yielding in compression. Subsequent cycle achieved the tensile strength of 1.4 Hy at a peak displacement of $4 \delta \mathrm{y}$. The corresponding compression strength was $-1.28 \mathrm{Hy}$ at $-4 \delta y$. However, the elastic stiffness during both unloading and reloading shows a clear decrease due to the progress of local bucking.

The envelope of the hysteretic curves for both specimens is shown in Fig. 5. It can be seen that the envelope of specimen 2 is generally above that of specimen 1 . This is due to the fact that larger strains in the yielding part would mean more strain hardening and also possibly more local buckling of the core. 


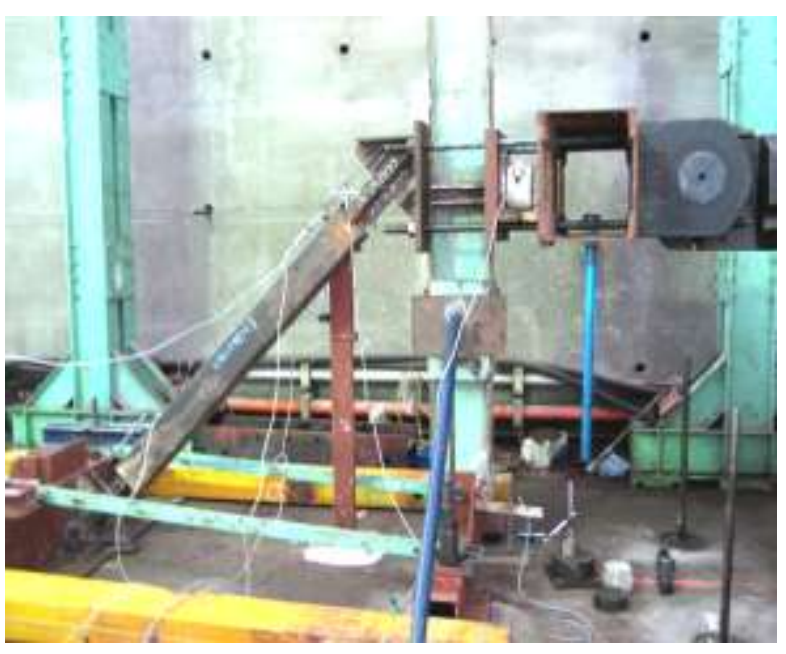

Fig. 3 Photo of test setup

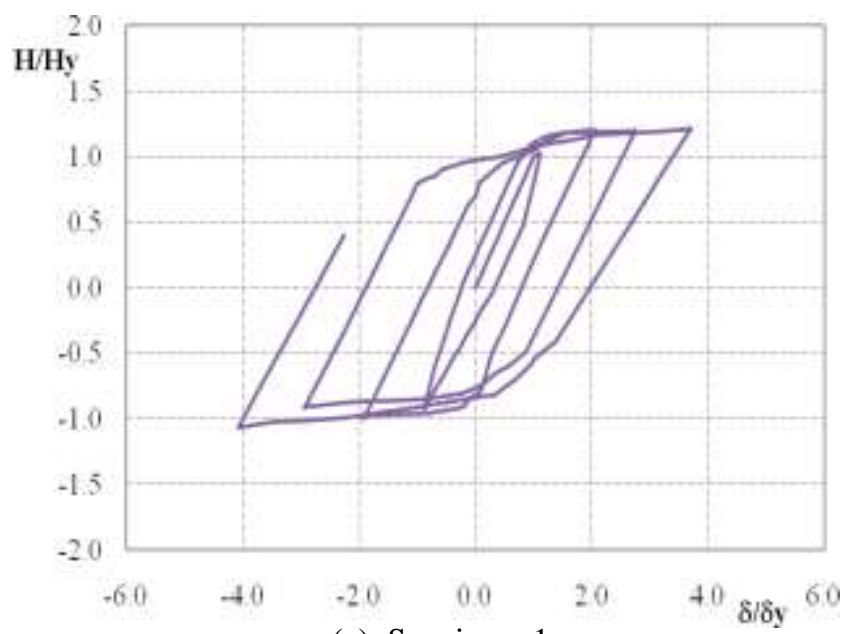

(a) Specimen 1

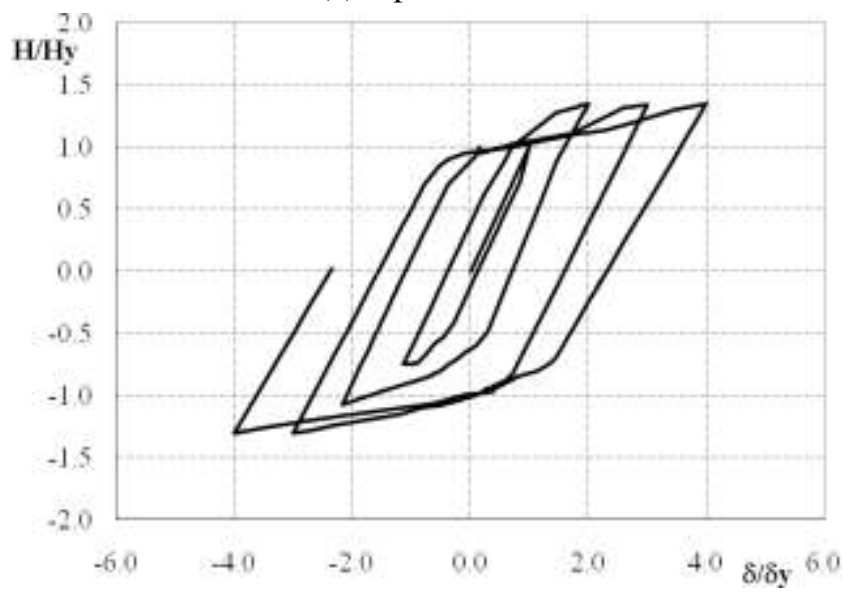

(b) Specimen 2

Fig. 4 Non-dimensional Load-displacement curves

\section{Summary and Conclusions}

Experimental study was carried out on two subassemblages with Buckling Restrained Braces. The two specimens had different lengths of the yielding parts. Results indicate that although the elastic stiffness of a BRB can be easily calculated, its response to cyclilc loading is not easy to predict. Reducing the core length gives increased stiffness and strength also leasing to increase energy dissipation. However, in the larger cycles degradation of stiffness occurs which may be detrimental to the objective of controlling storey drifts. Further tests may be needed to enable better prediction of the cyclic response so that desired responses can be achieved.

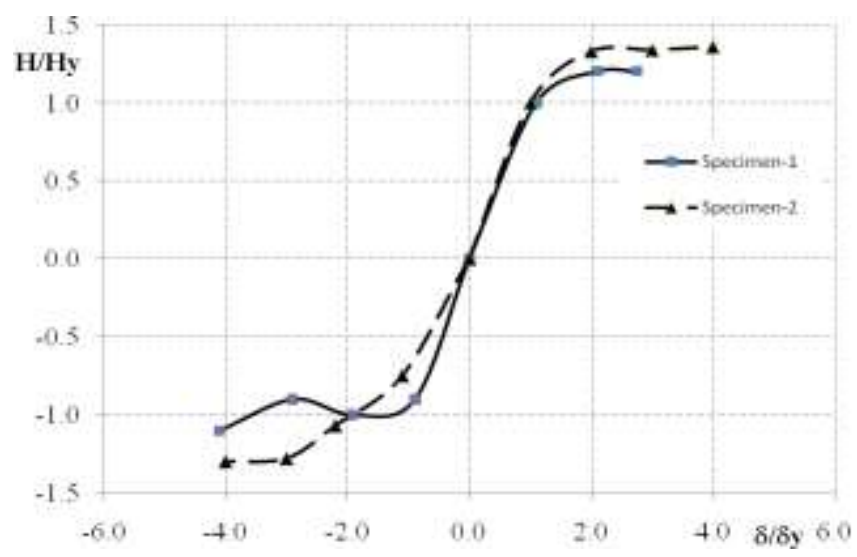

Fig. 5 Non-dimensional Load-deformation Envelopes

\section{References}

[1] G. Ravi Kumar, V. Kalyanaraman and S R Satish Kumar (2007) "Behavior of frames with non-buckling bracing under earthquakes", Journal of Constructional Steel Research, Elsevier, Vol. 63, pp. 254262.

[2] M. Iwata, T. Kato and A. Wada (2000) "Buckling-Restrained Braces as Hysteretic Dampers." Proc.STESSA 2000 Conference, Montreal, Canada, August 2000. Rotterdam, Balkema, 2000: 33-38.

[3] R. Tremblay, R. Neville and R. DeVall (2006), "Seismic performance and testing of bucking restrained bracing systems", Canadian Journal of Civil Engineering, 33 (2), 183-198, 10.1139/105-103.

[4] M. Masoud, G. Ali, P. Z. Amir and R. S. Hamid (2011). "Experimental optimization studies on steel core lengths in buckling restrained braces", Journal of Constructional Steel Research, 67(8):1244-1253.

[5] A. R. T. Seyyed, R. M. Seyyed and H. Abdollah (2014)“Experimental and numerical developing of reduced length buckling-restrained braces", Engineering Structures 10/2014; 77:143-160.

About Author (s)

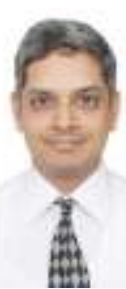

[Dr. S. R. Satish Kumar is currently Professor of Structural (Civil) Engineering at IIT Madras, Chennai, India. He areas of interest are Design of Steel Structures and Earthquake Resistant Design. 